\title{
A decrease in ubiquitination and resulting prolonged life-span of KIT underlies the KIT overexpression-mediated imatinib resistance of KIT mutation-driven canine mast cell tumor cells
}

\author{
MASATO KOBAYASHI, SHIORI KUROKI, SENA KURITA, RYO MIYAMOTO, \\ HIROYUKI TANI, KYOICHI TAMURA and MAKOTO BONKOBARA
}

Department of Veterinary Clinical Pathology, Nippon Veterinary and Life Science University, Kyonan-cho, Musashino-shi, Tokyo 180-8602, Japan

Received March 24, 2017; Accepted July 19, 2017

DOI: $10.3892 /$ or.2017.5865

\begin{abstract}
Overexpression of KIT is one of the mechanisms that contributes to imatinib resistance in KIT mutation-driven tumors. Here, the mechanism underlying this overexpression of KIT was investigated using an imatinib-sensitive canine mast cell tumor (MCT) line CoMS, which has an activating mutation in KIT exon 11. A KIT-overexpressing imatinib-resistant subline, rCoMS1, was generated from CoMS cells by their continuous exposure to increasing concentrations of imatinib. Neither a secondary mutation nor upregulated transcription of KIT was detected in rCoMS1 cells. A decrease in KIT ubiquitination, a prolonged KIT life-span, and KIT overexpression were found in rCoMS1 cells. These events were suppressed by withdrawal of imatinib and were re-induced by re-treatment with imatinib. These findings suggest that imatinib elicited overexpression of KIT via suppression of its ubiquitination. These results also indicated that imatinib-induced overexpression of KIT in rCoMS1 cells was not a permanently acquired feature but was a reversible response of the cells. Moreover, the pan deubiquitinating enzyme inhibitor PR619 prevented imatinib induction of KIT overexpression, suggesting that the imatinib-induced decrease in KIT ubiquitination could be mediated by upregulation and/or activation of deubiquitinating enzyme(s). It may be possible that a similar mechanism of KIT overexpression underlies the acquisition of imatinib resistance in some human tumors that are driven by KIT mutation.
\end{abstract}

\section{Introduction}

Imatinib is a small molecule kinase inhibitor with potent activity toward tumors whose growth is driven by mutations

Correspondence to: Professor Makoto Bonkobara, Department of Veterinary Clinical Pathology, Nippon Veterinary and Life Science University, 1-7-1 Kyonan-cho, Musashino-shi, Tokyo 180-8602, Japan E-mail: bonkobara@nvlu.ac.jp

Key words: canine mast cell tumor, imatinib, KIT, overexpression, resistance, ubiquitination in KIT, including gastrointestinal stromal tumor (GIST) (1) and certain subsets of mastocytosis (2) and melanoma (3). Despite their excellent initial response to imatinib, these tumors are generally not cured by this drug due to the acquisition of resistance. The mechanism of the resistance of KIT mutation-driven tumors to imatinib has been extensively studied in GIST; however, it is still not well understood. Although acquisition of secondary mutations in KIT is a major mechanism of the acquired resistance to imatinib, there are patients whose tumor does not have a secondary mutation in $\operatorname{KIT}(4,5)$. The mechanisms of the acquired resistance in these patients are heterogeneous and overexpression of KIT is one of the factors that contributes to imatinib resistance in such patients (6). KIT overexpression could be caused by mechanisms such as genomic amplification $(7,8)$ and upregulation of KIT transcription by overexpression of p55PIK, an isoform of phosphoinositide 3-kinase (9); however, the underlying mechanism of KIT overexpression is still unclear.

Similar to their development in humans, KIT mutation-driven tumors spontaneously develop in dogs (10-12). The neoplastic proliferation of mast cells in dogs, referred to as a mast cell tumor (MCT), is one of the most common tumors in this species (13), and $30 \%$ of all MCT cases and $70 \%$ of aggressive types of MCT possess an activating mutation in KIT (14). Canine MCT with KIT mutations respond well to kinase inhibitors including imatinib; however, they are usually not cured and eventually acquire resistance to the kinase inhibitors $(14,15)$. One of the mechanisms by which canine MCTs acquire resistance to imatinib could be by the generation of secondary mutations in KIT (16); however, similar to the case in human GIST, there are imatinib-resistant cases without such mutations in KIT.

Due to its potential ability to acquire resistance to imatinib, canine MCT is considered a valuable spontaneous model for translational research in investigation of the mechanisms, and in overcoming strategies of imatinib resistance in KIT mutation-driven human tumors. A canine MCT cell line, CoMS, has been established from a dog with oral mucosa MCT (17). CoMS cells have an imatinib-sensitive activating mutation in the juxtamembrane domain of KIT (KIT exon 11, c.1720_1772+1dup). In the present study, we generated a 
KIT-overexpressing imatinib-resistant subline from CoMS cells and investigated the mechanisms underlying KIT overexpression that caused imatinib resistance.

\section{Materials and methods}

Cell line. The canine MCT cell line CoMS was kindly provided by Dr Takagi (University of Hokkaido, Hokkaido, Japan). Madin-Darby canine kidney (MDCK) cells were purchased from the American Type Culture Collection (Manassas, VA, USA). CoMS cells were cultured in RPMI-1640 medium (Life Technologies, Carlsbad, CA, USA) supplemented with $10 \%$ fetal calf serum (Nippon Bio-Supply, Tokyo, Japan), $50 \mathrm{U} / \mathrm{ml}$ penicillin (Life Technologies), and $50 \mu \mathrm{g} / \mathrm{ml}$ streptomycin (Life Technologies) (cRPMI) in a humidified incubator at $37^{\circ} \mathrm{C}$ under $5 \% \mathrm{CO}_{2}$. MDCK cells were cultured in Dulbecco's modified Eagle's medium (Life Technologies) supplemented with the same additives, under the same conditions.

Generation of an imatinib-resistant CoMS subline. CoMS cells were exposed to increasing concentrations of imatinib (LC Laboratories, Woburn, MA, USA), starting with a concentration of $0.02 \mu \mathrm{M}$ and then gradually increasing up to a concentration of $1 \mu \mathrm{M}$ over two months. The cells that grew logarithmically in the presence of $1 \mu \mathrm{M}$ of imatinib were named rCoMS1 cells and were maintained in cRPMI supplemented with $1 \mu \mathrm{M}$ of imatinib.

Cell growth inhibition assay. CoMS and rCoMS1 cells suspended in cRPMI were seeded in 96-well plates $\left(2 \times 10^{4}\right.$ cells/well) and treated with various concentrations of imatinib $(0-100 \mu \mathrm{M})$ for $24 \mathrm{~h}$. Cell viability was then measured using a WST-1 cell proliferation assay kit (Takara, Otsu, Japan). The half maximal inhibitory concentration $\left(\mathrm{IC}_{50}\right)$ of imatinib was calculated using the GraphPad Prism software program (GraphPad Software, San Diego, CA, USA).

Flow cytometric analysis of KIT. Cellular expression of KIT was analyzed using flow cytometry. For detection of cell surface KIT, CoMS and rCoMS1 cells were fixed with 4\% paraformaldehyde for $20 \mathrm{~min}$ and washed twice with PBS-2\% BSA. As a negative control, MDCK cells were also subjected to the same assay. The cells were stained with phycoerythrin (PE)-conjugated monoclonal rat anti-mouse KIT (ACK45) or PE-conjugated isotype-matched control IgG (rat IgG2b, א) (both from BD Biosciences, San Diego, CA, USA) for $1 \mathrm{~h}$ in PBS-2\% BSA. The cells were then analyzed by flow cytometry using a FACSCalibur (BD Biosciences). For detection of total (cell surface and intracellular) KIT, PBS-2\% BSA was replaced with permeabilization buffer (eBioscience, San Diego, CA, USA) and the cellular expression of KIT was analyzed using flow cytometry in the same manner.

Detection of KIT and phosphorylated KIT by western blot analysis. CoMS and rCoMS1 cells suspended in cRPMI were seeded in 6-well plates $\left(5 \times 10^{5}\right.$ cells/well $)$ and treated with different concentrations of imatinib $(0-10 \mu \mathrm{M})$ for $4 \mathrm{~h}$. The cells were then lysed, and aliquots were subjected to western blot analysis using the following antibodies: polyclonal goat anti-human KIT (C-14) (Santa Cruz Biotechnology, Santa Cruz, CA, USA), monoclonal rabbit anti-human phospho-KIT (Tyr703, D12E12) (Cell Signaling, Danvers, MA, USA), or polyclonal goat anti-human GAPDH (Santa Cruz Biotechnology), followed by biotin-conjugated rabbit anti-goat or goat anti-rabbit IgG (Life Technologies). After incubation of the membranes with peroxidase-conjugated streptavidin, immunoreactive bands were visualised using an enhanced chemiluminescence system (GE Healthcare, Chalfont, UK) and the LAS-4000 (Fujifilm, Tokyo, Japan).

Semi-quantitative RT-PCR analysis of KIT expression. Total RNA extracted from CoMS and rCoMS1 cells was reversetranscribed into cDNA and subjected to PCR amplification using Platinum Taq DNA Polymerase (Life Technologies) and a primer set for canine KIT: 5' primer, 5'-GCTGGTCCGCTG CCCTCTGA-3'; 3' primer, 5'-GGCGTAACACATGAACA CTCCA-3'. After PCR amplification for 22 cycles, the products (10 $\mu \mathrm{l}$ aliquot) were size-fractionated on a $1.2 \%$ agarose gel and visualized with ethidium bromide staining and the LAS-500 (Fujifilm). Band intensities were semi-quantified using ImageQuant TL software (Fujifilm).

Cycloheximide (CHX) chase assay. CoMS and rCoMS1 cells seeded in a 6 -well plate $\left(5 \times 10^{5}\right.$ cells/well) were incubated with $50 \mu \mathrm{g} / \mathrm{ml}$ of CHX (Life Technologies) for 0, 30, 60 and $120 \mathrm{~min}$. After culture, these cells were washed twice with PBS and lysed. The cell lysates were then subjected to western blot analysis for detection of KIT and GAPDH.

Analysis of the ubiquitination status of KIT. CoMS and rCoMS1 cells were lysed and the KIT protein was immunoprecipitated using Protein G-agarose beads (Life Technologies) bound with polyclonal rabbit anti-human KIT (Dako Corp., Carpinteria, CA, USA). The immunoprecipitated proteins were subjected to western blot analysis using polyclonal rabbit anti-ubiquitin antibody (Abcam, Cambridge, MA, USA) or polyclonal goat anti-human KIT (C-14) (Santa Cruz Biotechnology).

Analysis of KIT status in rCoMS1 cells following imatinib withdrawal and subsequent imatinib re-treatment. $\mathrm{rCoMS1}$ cells that were maintained in cRPMI supplemented with $1 \mu \mathrm{M}$ of imatinib were transferred into imatinib-free cRPMI and culture was continued for a further $0,7,14$ and 21 days. The rCoMS1 cells that were cultured in imatinib-free cRPMI for 14 days (rCoMS1-IM14d cells) were further cultured in cRPMI supplemented with $1 \mu \mathrm{M}$ of imatinib for $0,6,24,48$ and $72 \mathrm{~h}$. The expression levels of KIT in rCoMS1 cells under these conditions were examined by western blot analysis. The rCoMS1-IM14d cells and the cells that had been re-treated with imatinib for $48 \mathrm{~h}$ (rCoMS1-IM14d+IM48h cells) were subjected to a CHX chase assay, ubiquitination status analysis, KIT and phosphorylated KIT analysis, and cell growth inhibition assay using the methods described above. The rCoMS1-IM14d cells were also culture in cRPMI with imatinib ( 0 or $1 \mu \mathrm{M})$ and the pan deubiquitinating enzyme inhibitor PR619 (Sigma-Aldrich, St. Louis, MO, USA) $(0$ or $20 \mu \mathrm{M})$ for $48 \mathrm{~h}$, following which the expression level of KIT was examined using western blot analysis. 
A

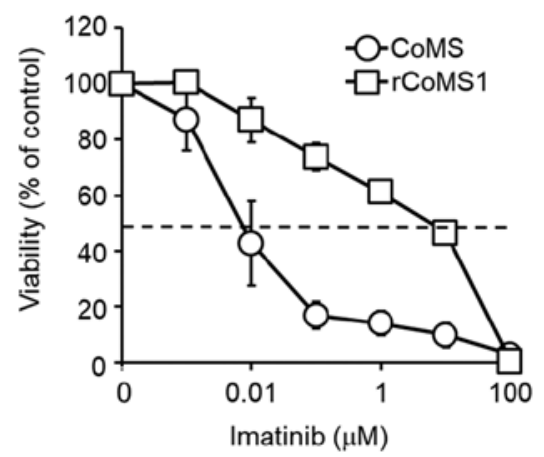

B

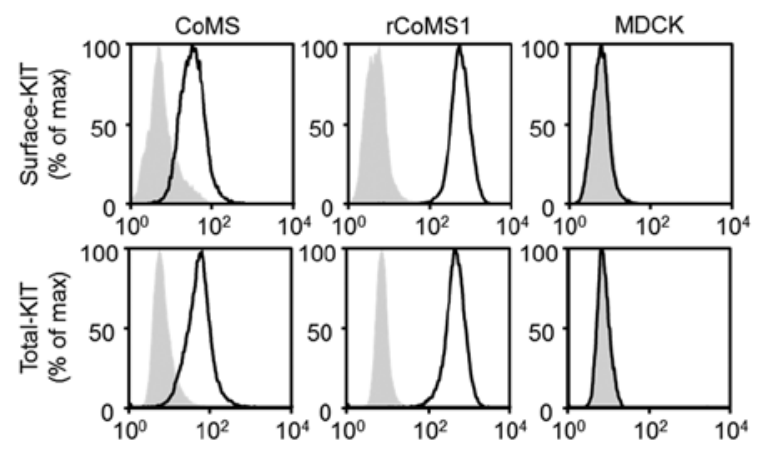

C
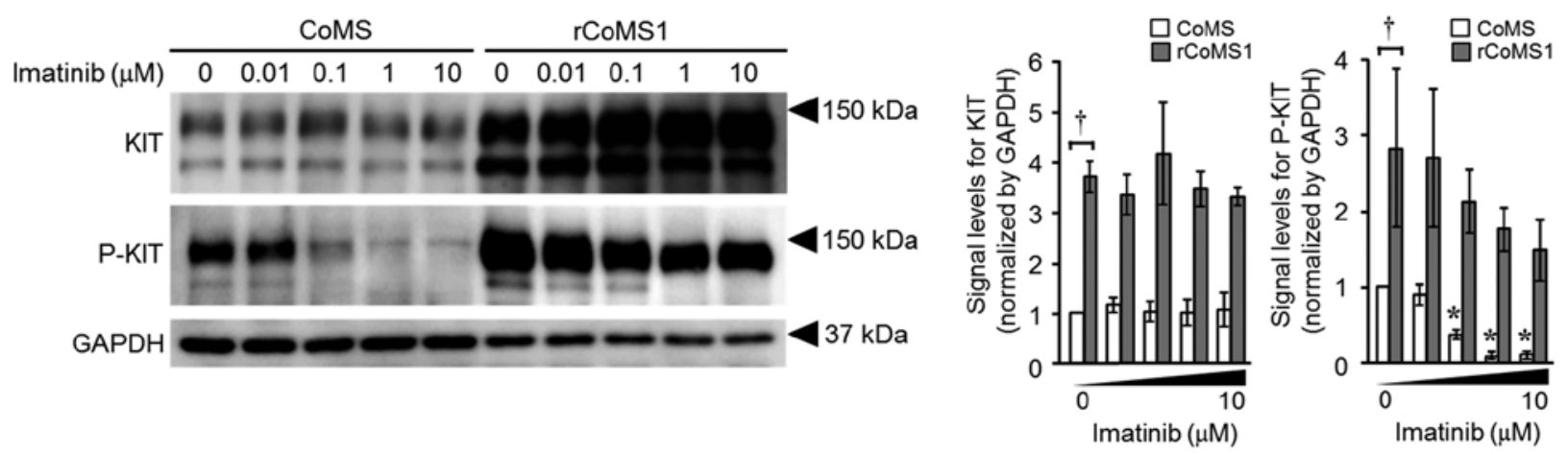

Figure 1. Development of a KIT-overexpressing imatinib-resistant rCoMS1 cell line. (A) Effect of imatinib on the growth of CoMS and rCoMS1 cells. Cell viability was expressed as a percentage of carrier control-treated ( $0 \mu \mathrm{M}$ imatinib; dimethyl sulfoxide, final concentration of $0.01 \%)$ cells. Data are expressed as means \pm SD $(n=4)$. (B) Flow cytometric analysis of KIT in CoMS, rCoMS1, and negative control (MDCK) cells. Upper and lower graphs show the data of non-permeabilized (surface-KIT) and permeabilized (total-KIT) cells, respectively. Shaded and black line histograms represent staining with control (isotypematched IgG) and anti-KIT antibody, respectively. (C) Western blot analysis for KIT (KIT) and phosphorylated KIT (P-KIT) using CoMS and rCoMS1 cells. The cells were treated with the indicated concentration of imatinib $(0-10 \mu \mathrm{M})$ for $4 \mathrm{~h}$. GAPDH was used as an internal control. Left panel, representative western blot image of three independent experiments. Right panel, semi-quantification of the signal levels of KIT (left graph) and phosphorylated KIT (right graph) normalized to GAPDH is shown (white bars, CoMS; grey bars, rCoMS1). The normalized signal level of KIT and P-KIT in CoMS cells in the absence of imatinib $(0 \mu \mathrm{M})$ was set at 1.0. Data are expressed as means $\pm \mathrm{SD}(\mathrm{n}=3)$. ${ }^{\dagger}$ Significant difference between CoMS vs. rCoMS1 cells at $0 \mu \mathrm{M}$ imatinib (P<0.01). "Significant difference vs. $0 \mu \mathrm{M}$ imatinib $(\mathrm{P}<0.01)$

Statistical analysis. Statistical analysis was performed using an unpaired two-tailed Student's $t$-test. Significance was accepted at $\mathrm{P}<0.05$.

\section{Results}

Overexpression of activated KIT in the rCoMS1 imatinibresistant cell line. A canine MCT cell line, CoMS, has been used in this study because canine MCT can be a spontaneous model for translational research of imatinib resistance in KIT mutation-driven human tumors and CoMS cells have an imatinib-sensitive activating mutation in KIT. An imatinibresistant CoMS cell line, rCoMS1, was generated by the continuous exposure of CoMS cells to increasing concentrations of imatinib (Fig. 1A). The calculated $\mathrm{IC}_{50}$ value of imatinib for rCoMS1 cells was $>200$-fold higher than that for CoMS cells $\left(\mathrm{IC}_{50}\right.$, CoMS, $0.038 \pm 0.022 \mu \mathrm{M}$; rCoMS1, $9.0 \pm 2.7 \mu \mathrm{M} ; \mathrm{P}<0.01)$. KIT was mostly expressed on the cell surface of both CoMS and rCoMS1 cells but the expression level of KIT was much higher on rCoMS1 cells compared with CoMS cells (Fig. 1B). The entire nucleotide sequence of KIT was examined in CoMS and rCoMS1 cells but no mutation other than the original mutation of c.1720_1772+1dup was found in both cell lines (data not shown).
In western blot analysis of KIT, the signals of both KIT and phosphorylated KIT were much higher in rCoMS1 cells compared with those in CoMS cells under the non-treated condition (Fig. 1C, imatinib $0 \mu \mathrm{M}$ ). The phosphorylation of KIT was concentration dependently suppressed by imatinib in CoMS cells and strong suppression of phosphorylation was detected following treatment with $0.1 \mu \mathrm{M}$ or more of imatinib (Fig. 1C). In contrast, there was only a small imatinib-induced decrease in KIT phosphorylation in rCoMS1 cells and a high level of KIT phosphorylation was maintained under treatment with the maximum concentration of imatinib $(10 \mu \mathrm{M})$ (Fig. 1C).

Prolonged life-span of KIT caused by a decrease in its ubiquitination in rCoMS1 cells. To examine if overexpression of KIT in rCoMS1 cells is due to an increase in the level of the KIT transcripts, the expression level of $K I T$ mRNA was evaluated. No difference in the expression level of KIT mRNA was found between CoMS and rCoMS1 cells (Fig. 2A). We then examined the difference in KIT protein life-span between CoMS and rCoMS1 cells. For this purpose, a CHX chase assay, which monitors the time course of protein degradation after translation is arrested by CHX treatment, was employed (Fig. 2B). In CoMS cells, the amount of KIT was time-dependently 
A

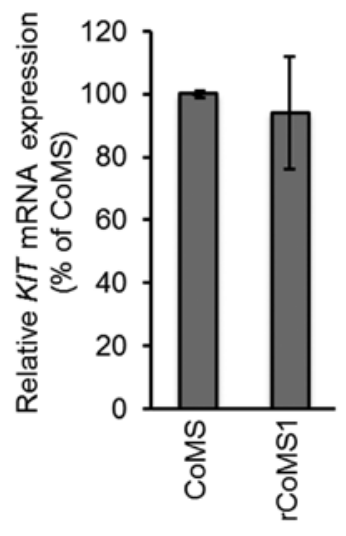

C

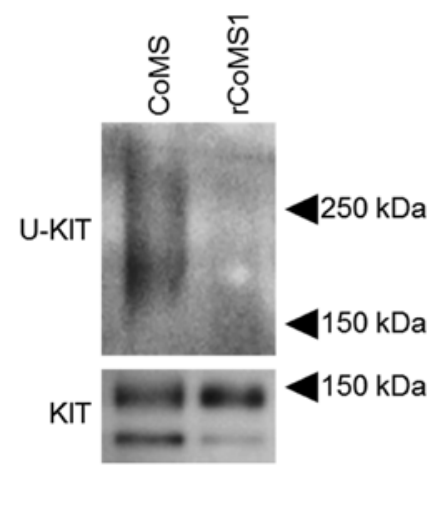

B
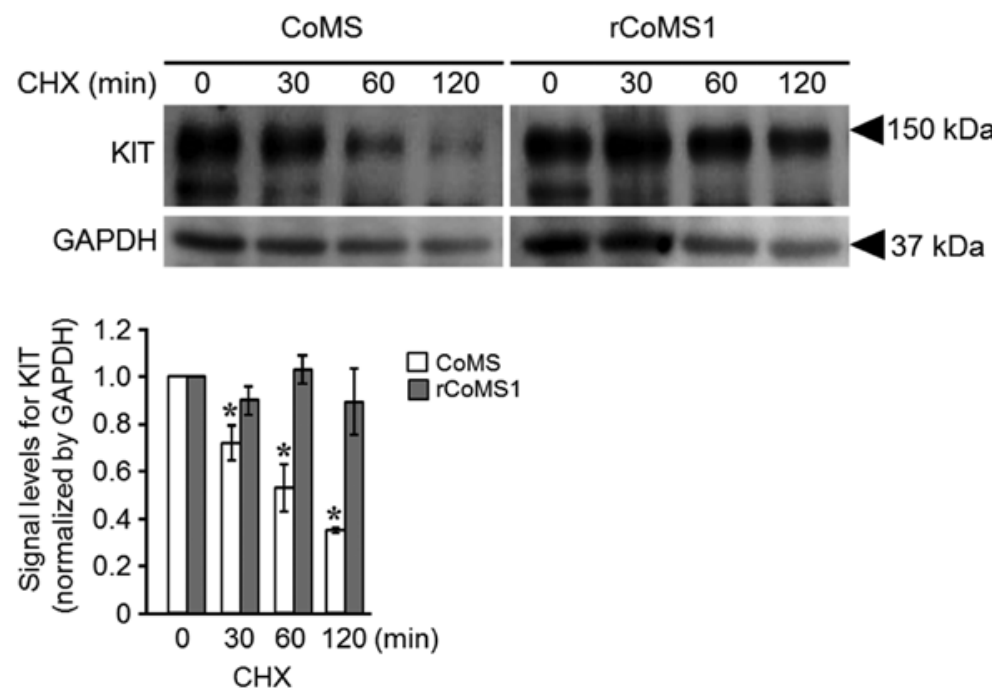

Figure 2. Analysis of KIT mRNA expression, life-span and ubiquitination status in CoMS and rCoMS1 cells. (A) Semi-quantitative RT-PCR analysis of KIT mRNA expression. The expression level of KIT mRNA in rCoMS1 cells was expressed as a percentage of that in CoMS cells. Data are expressed as means \pm SD $(\mathrm{n}=4)$. No statistically significant difference in KIT expression was found between these cell lines. (B) Cycloheximide (CHX) chase assay for KIT using CoMS and rCoMS1 cells. CHX $(50 \mu \mathrm{g} / \mathrm{ml})$ was used to block protein synthesis. After treatment of the cells with $\mathrm{CHX}$ for the indicated time (0-120 min), the cells were subjected to western blot analysis for detection of KIT and control GAPDH. Upper panel, representative western blot image of three independent experiments. Lower panel, semi-quantification of the signal levels of KIT normalized to GAPDH is shown (white bars, CoMS; grey bars, rCoMS1). The normalized signal level of KIT in cells at 0 min CHX was set at 1.0. Data are expressed as means \pm SD ( $\mathrm{n}=3$ ). * Significant difference vs. 0 min (P<0.01). (C) Analysis of the ubiquitination status of KIT in CoMS and rCoMS1 cells. KIT was immunoprecipitated from the cells and subjected to western blot analysis for ubiquitin (U-KIT) or KIT (KIT). A ubiquitin-positive smear band was detected in the lane of CoMS cells but was barely detected in that of rCoMS1 cells. The data are representative of three independent experiments.

decreased after treatment of the cells with CHX and KIT was only weakly detected after treatment of $60 \mathrm{~min}$. In contrast, there was only a small decrease in the level of KIT after treatment of rCoMS1 cells with CHX, and a high level of KIT remained at 120 min of CHX treatment, indicating a prolonged life-span of KIT in rCoMS1 cells. Since this prolonged life-span could be caused by retardation of KIT degradation, the ubiquitination status of KIT in CoMS and rCoMS1 cells was examined using western blot analysis (Fig. 2C). Ubiquitinated KIT was clearly detected in CoMS cells, while it was barely detected in rCoMS1 cells (Fig. 2C).

Imatinib-induced KIT deubiquitination-mediated reversible overexpression of KIT in rCoMS1 cells. To clarify if the prolonged life-span of KIT in rCoMS1 cells was caused in response to imatinib, we examined the changes in the KIT expression level in rCoMS1 cells under conditions of imatinib-withdrawal and subsequent imatinib re-treatment (Fig. 3). The expression level of KIT in rCoMS1 cells gradually decreased over time after removal of imatinib from the culture medium and a strong decrease was noted on day 14 and thereafter (Fig. 3A, upper panel). The rCoMS1 cells that had been cultured for 14 days without imatinib (rCoMS1-IM14d cells) were then re-treated with imatinib (Fig. 3A, lower panel). KIT expression increased again at $24 \mathrm{~h}$, reaching a maximum at $48 \mathrm{~h}$ after re-treatment of the rCoMS1-IM14d cells with imatinib. In contrast, no difference in the expression level of KIT mRNA was found between rCoMS1 cells, rCoMS1-IM14d cells and rCoMS1-IM14d cells that had been re-treated with imatinib for $48 \mathrm{~h}$ (rCoMS1-IM14d+IM48h cells) (Fig. 3B). To examine the difference in the life-span of KIT between rCoMS1-IM14d cells and rCoMS1-IM14d+IM48h cells, a CHX chase assay was performed (Fig. 3C). After protein synthesis was inhibited by treatment with $\mathrm{CHX}$, the KIT in 
A
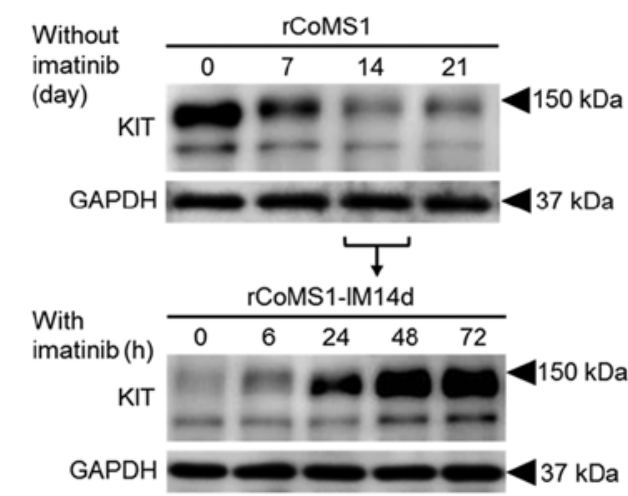

C
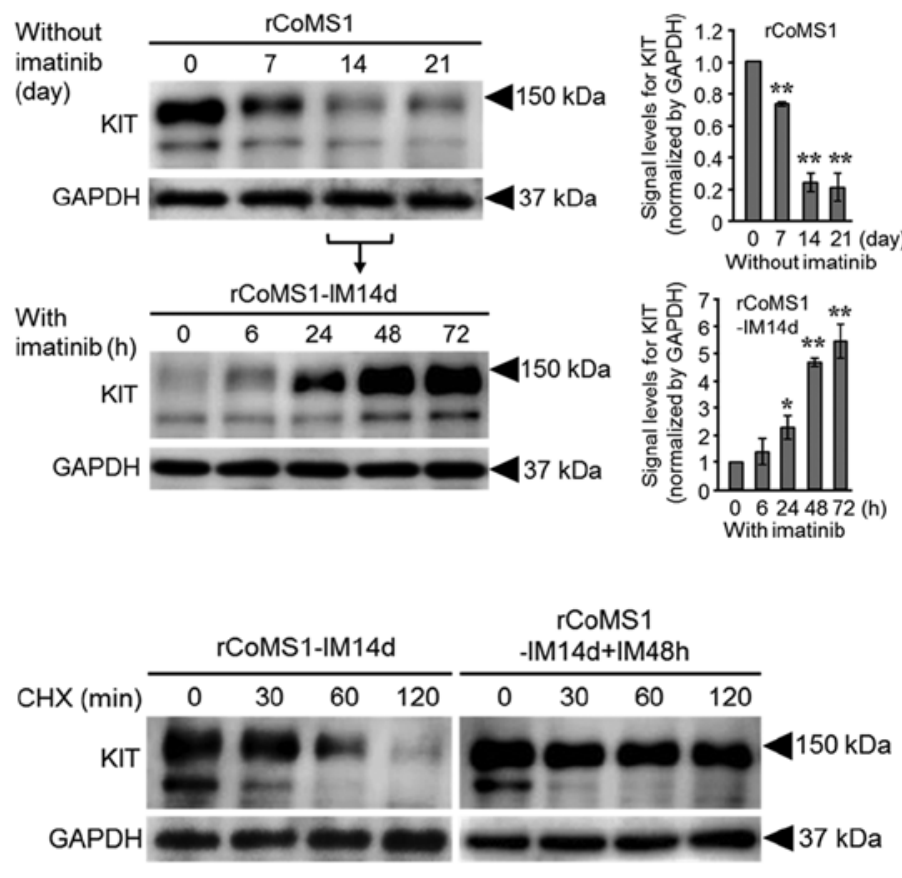
Without imatinib

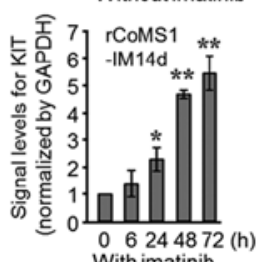

B

E
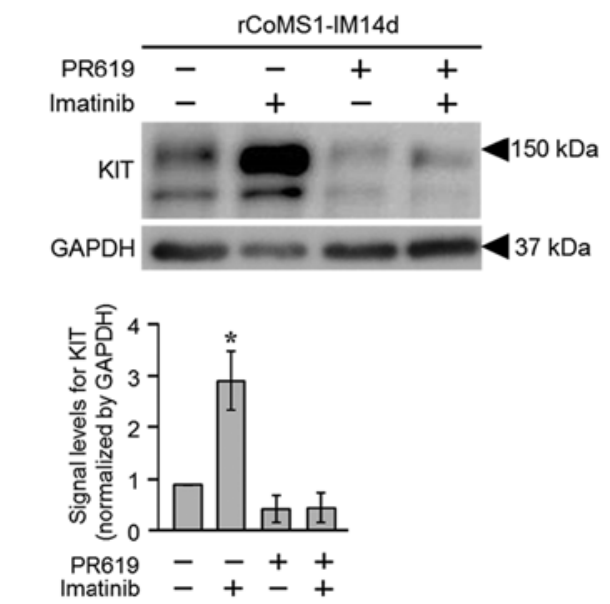

Figure 3. Effects of imatinib-withdrawal and subsequent imatinib re-treatment on KIT expression and ubiquitination status in rCoMS1 cells. (A) Changes in the expression level of KIT in rCoMS1 cells under imatinib-withdrawal and subsequent imatinib re-treatment. rCoMS1 cells were cultured for 0-21 days without imatinib (upper panel). rCoMS1 cells cultured for 14 days in the absence of imatinib (rCoMS1-IM14d) were then re-treated with $1 \mu \mathrm{M}$ imatinib for 0-72 $\mathrm{h}$ (lower panel). Expression of KIT in rCoMS1 cells under these conditions was examined by western blot analysis. GAPDH was blotted as an internal control. Left panel, representative western blot image of three independent experiments. Right panel, semi-quantification of the signal levels of KIT normalized to GAPDH is shown. The normalized signal level of KIT on day 0 (rCoMS1) and at $0 \mathrm{~h}\left(\mathrm{rCoMS1-IM14d)}\right.$ ) was set at 1.0. Data are expressed as means $\pm \mathrm{SD}(\mathrm{n}=3)$. ${ }^{*} \mathrm{P}<0.05$, ${ }^{* *} \mathrm{P}<0.01$. Significant difference vs. day 0 (rCoMS1) or $0 \mathrm{~h}$ (rCoMS1-IM14d). (B) Semi-quantitative RT-PCR analysis of KIT mRNA expression. The expression levels of KIT mRNA in rCoMS1-IM14d cells and rCoMS1-IM14d+IM48h cells were expressed as a percentage of that in rCoMS1 cells. Data are expressed as means $\pm \mathrm{SD}(\mathrm{n}=4)$. No statistically significant difference in KIT expression was found between these cell lines. (C) Cycloheximide (CHX) chase assay for KIT expression in rCoMS1-IM14d cells and in the same cells that were further cultured for $48 \mathrm{~h}$ in the presence of $1 \mu \mathrm{M}$ imatinib (rCoMS1-IM14d+IM48h). After treatment of the cells with CHX (50 $\mu \mathrm{g} / \mathrm{ml})$ for the indicated time $(0-120 \mathrm{~min})$, the cells were subjected to western blot analysis for detection of KIT and control GAPDH. Upper panel, representative western blot image of three independent experiments. Lower panel, semi-quantification of the signal levels of KIT normalized to GAPDH is shown (white bars, rCoMS1-IM14d; grey bars, rCoMS1-IM14d+IM48h). The normalized signal level of KIT in CHX 0 min cells was set at 1.0. Data are expressed as means $\pm \mathrm{SD}(\mathrm{n}=3)$. ${ }^{*}$ Significant difference vs. 0 min $(\mathrm{P}<0.01)$. (D) Western blot analysis of the ubiquitination status of KIT (U-KIT) in rCoMS1-IM14d and rCoMS1-IM14d+IM48h cells. An ubiquitin-positive smear band was detected in the lane of rCoMS1-IM14d cells but was barely detected in that of rCoMS1-IM14d+IM48h cells. The data are representative of three independent experiments. (E) Effect of the pan deubiquitinating enzyme inhibitor PR619 $(20 \mu \mathrm{M})$ on the expression status of KIT in rCoMS1-IM14d cells cultured in the presence or absence of imatinib (1 $\mu \mathrm{M})$ for $48 \mathrm{~h}$. KIT and control GAPDH were detected by western blot analysis. Upper panel, representative western blot image of three independent experiments. Lower panel, semi-quantification of the signal levels of KIT normalized to GAPDH is shown. The normalized signal level of KIT in the condition of imatinib (-)/PR619 (-) was set at 1.0. Data are expressed as means \pm SD (n=3). . Significant difference vs. imatinib (-)/PR619 (-) $(\mathrm{P}<0.01)$.

the rCoMS1-IM14d cells was degraded in a time-dependent manner, while little KIT degradation was observed over time in the rCoMS1-IM14d+IM48h cells (Fig. 3C). Fig. 3D shows the ubiquitination status of KIT in rCoMS1-IM14d and rCoMS1-IM14d+IM48h cells. Ubiquitinated KIT was clearly detected in the rCoMS1-IM14d cells, while it was barely detected in the rCoMS1-IM14d+IM48h cells. Since dysregulation of deubiquitinating enzyme(s) is one potential mechanism that might impair ubiquitination-mediated downregulation of receptor tyrosine kinases $(18,19)$, the effects of the deubiquitinating enzyme inhibitor PR619 on the induction of KIT overexpression by imatinib in rCoMS1-IM14d cells was examined. As shown in Fig. 3E, PR619 strongly suppressed imatinib-induced KIT overexpression in rCoMS1-IM14d cells.

Linkage of recovery of imatinib sensitivity and re-acquirement of resistance to imatinib of rCoMSI cells to KIT expression status. To confirm that the sensitivity of rCoMS1 cells to imatinib was linked to the expression status of KIT, we examined the effects of imatinib on the growth of rCoMS1-IM14d and rCoMS1-IM14d+IM48h cells. As shown in Fig. 4A, 
A

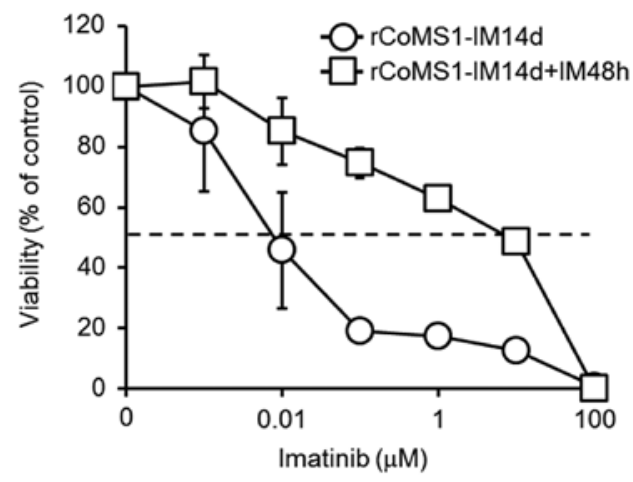

B
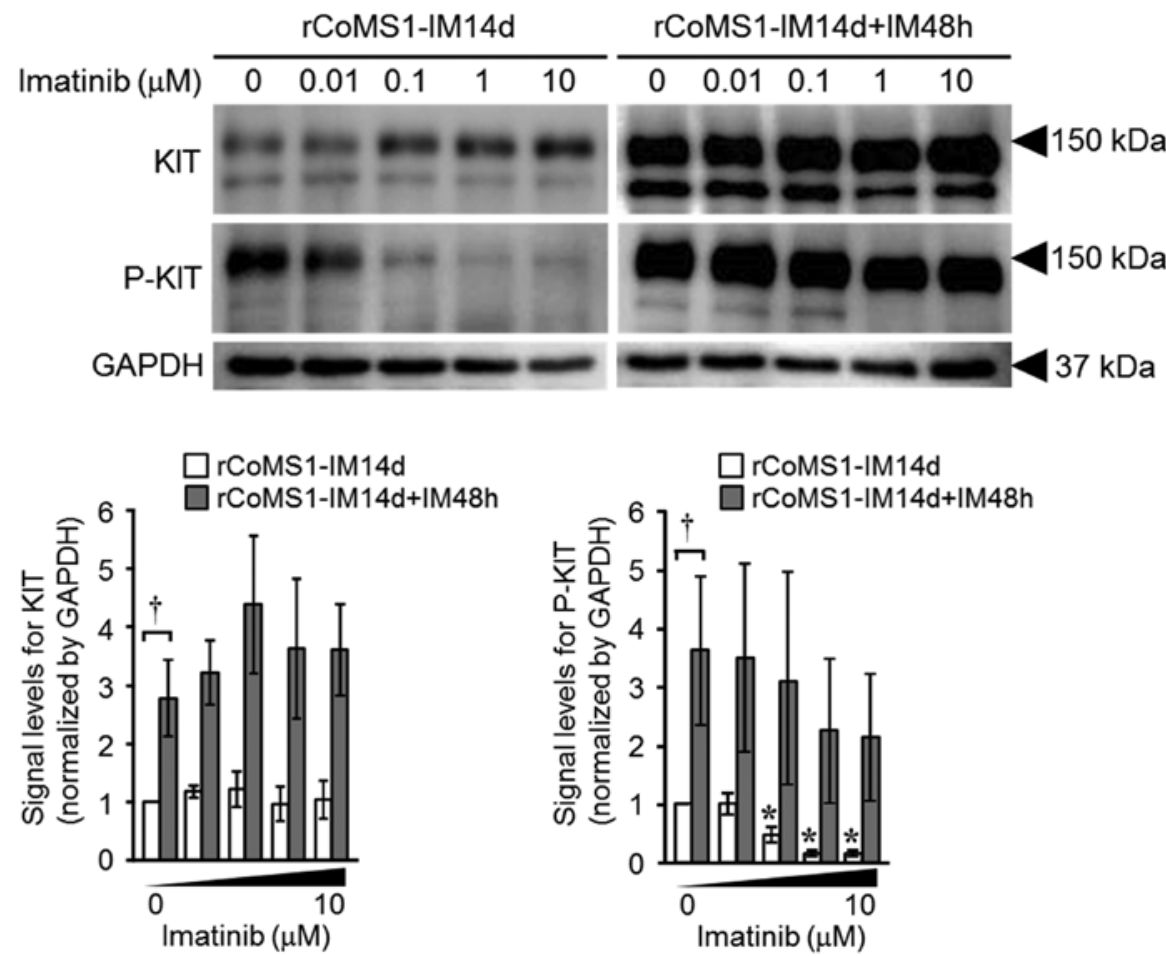

Figure 4. The expression status of KIT is linked to the resistance of rCoMS1 cells to imatinib. (A) Effect of imatinib on the growth of rCoMS1-IM14d and rCoMS1-IM14d+IM48h cells. Cell viability was expressed as a percentage of carrier control-treated (0 $\mu \mathrm{M}$ imatinib; dimethyl sulfoxide, final concentration of $0.01 \%$ ) cells. Data are expressed as means \pm SD $(n=4)$. (B) Western blot analysis of KIT (KIT) and phosphorylated KIT (P-KIT) in rCoMS1-IM14d and rCoMS1-IM14d+IM48h cells treated with the indicated concentration of imatinib (0-10 $\mu \mathrm{M})$ for $4 \mathrm{~h}$. GAPDH was used as an internal control. Upper panel, representative western blot image of three independent experiments. Lower panel, semi-quantification of the signal levels of KIT (left graph) and phosphorylated KIT (right graph) normalized to GAPDH is shown (white bars, rCoMS1-IM14d; grey bars, rCoMS1-IM14d+IM48h). The normalized signal level of KIT and P-KIT in rCoMS1-IM14d cells in the absence of imatinib $(0 \mu \mathrm{M})$ was set at 1.0 . Data are expressed as means \pm SD (n=3). ${ }^{\dagger}$ Significant difference for rCoMS1-IM14d vs. rCoMS1-IM14d+IM48h cells at $0 \mu \mathrm{M}$ imatinib $(\mathrm{P}<0.01)$. "Significant difference vs. $0 \mu \mathrm{M}$ imatinib $(\mathrm{P}<0.01)$.

growth of the rCoMS1-IM14d cells was efficiently inhibited by imatinib, while rCoMS1-IM14d+IM48h cells had re-acquired resistance to imatinib $\left(\mathrm{IC}_{50}:\right.$ rCoMS1-IM14d, $0.043 \pm 0.023 \mu \mathrm{M}$; rCoMS1-IM14d+IM48h, 9.45 $\pm 2.85 \mu \mathrm{M} ; \mathrm{P}<0.01)$. Consistent with these findings, imatinib efficiently suppressed the phosphorylation of KIT in rCoMS1-IM14d cells, while it failed to suppress the phosphorylation of the re-overexpressed KIT in rCoMS1-IM14d+IM48h cells (Fig. 4B).

\section{Discussion}

This is the first report to suggest that an imatinib-induced decrease in ubiquitination and a subsequent prolonged life-span of KIT underlies the overexpression of KIT that contributes to imatinib resistance of KIT mutation-driven tumors.

The role of KIT overexpression in the acquisition of resistance to imatinib in CoMS cells was supported by the findings that: i) a high level of KIT phosphorylation was maintained in the presence of imatinib; ii) the resistance of rCoMS1 cells to imatinib was clearly linked to changes in the expression status of KIT; and iii) no secondary mutation in KIT was identified in rCoMS1 cells. Analyses of the expression of KIT mRNA and the life-span of KIT, suggested that a prolonged KIT life-span rather than an increase in de novo synthesis caused the observed overexpression of KIT. Moreover, this prolonged life-span of KIT was caused by a decrease in KIT ubiquitination that would 
result in the retardation of its degradation in rCoMS1 cells. The decrease in KIT ubiquitination in rCoMS1 cells may also explain why KIT is overexpressed on the surface of rCoMS1 cells. Following activation, cell surface KIT is ubiquitinated and subsequently targeted for endocytosis and degradation in the lysosomes (20). Thus, a decrease in KIT ubiquitination and subsequent retarded degradation of KIT may result in its accumulation and overexpression on the surface of rCoMS1 cells.

Regarding the overexpression of KIT in rCoMS1 cells, we found that: i) KIT overexpression in rCoMS1 cells was decreased by withdrawal of imatinib (rCoMS1-IM14d cells) and KIT was re-overexpressed by re-treatment with imatinib (rCoMS1-IM14d+IM48h cells); ii) KIT life-span was shortened in rCoMS1-IM14d cells compared to rCoMS1 cells but its life-span increased again in rCoMS1-IM14d+IM48h cells; and iii) KIT was ubiquitinated in rCoMS1-IM14d cells but its ubiquitination level was markedly decreased in rCoMS1-IM14d+IM48h cells. These findings confirmed that the following series of events was induced in response to imatinib: a decrease in KIT ubiquitination, a prolongation of KIT life-span, and the resulting overexpression of KIT in rCoMS1 cells. These findings also indicated that imatinibinduced overexpression of KIT in rCoMS1 cells is not a permanently acquired feature but is a reversible response of the cells. Moreover, imatinib failed to induce overexpression of KIT in rCoMS1-IM14d cells in the presence of the pan deubiquitinating enzyme inhibitor PR619 (Fig. 3E). This finding suggested that the imatinib-induced decrease in KIT ubiquitination could be mediated by upregulation and/or activation of deubiquitinating enzyme(s). In the PR619 experiment, PR619 did not completely suppress KIT expression. The residual KIT could be an inactivated form of KIT since, although activated KIT undergoes ubiquitin-mediated degradation, inactivated KIT is degraded via an ubiquitinindependent process (21). Since a deubiquitinating enzyme specific for ubiquitinated KIT has not yet been identified, we were unable to confirm the role of such an enzyme in imatinib modulation of KIT by using a KIT-specific deubiquitinating enzyme inhibitor. However, in a recent study, it was shown that aberrantly activated USP8, an enzyme that deubiquitinates several receptor tyrosine kinases such as EGFR, Frizzled, ERBB, Met and Smoothened (18), induces the cell surface overexpression of the EGFR by switching the endocytic trafficking pathway of the EGFR from the endosome-lysosome degradation pathway to the recycling pathway that recycles EGFR back to the plasma membrane (19). Collectively, this finding and our results might suggest that upregulation and/or activation of a KIT deubiquitinating enzyme by imatinib could induce a similar pathway switching of KIT that induces cell surface overexpression of KIT on CoMS cells.

Dysregulation of ubiquitination pathways that underlies resistance to kinase inhibitors has also been reported in human melanomas (22). The ubiquitin ligase, RNF125, is downregulated in BRAF inhibitor-resistant melanomas, resulting in increased expression of its substrate, JAK1, with a concomitant increase in EGFR expression (22). This overexpression of JAK1 and EGFR was suggested to underlie the resistance of melanoma cells to BRAF inhibitors. In contrast with BRAF inhibitor-resistant melanomas, downregulation of an ubiquitin ligase such as $\mathrm{Cbl}$, which is an enzyme that can ubiquitinate KIT (23), would not be involved in imatinib resistance in rCoMS1 cells because: i) overexpression of KIT was clearly inhibited by a deubiquitinating enzyme inhibitor in rCoMS1-IM14d cells and, ii) overexpression of KIT due to downregulation of a ubiquitin ligase would not be prevented by a deubiquitinating enzyme inhibitor.

The development of KIT-overexpressing imatinib-resistant rCoMS1 cells required $>2$ months culture in the presence of imatinib, whereas imatinib-sensitive rCoMS1-IM14d cells re-overexpressed KIT and acquired resistance to imatinib in a short period ( 24-48 h) after re-treatment with imatinib. Although the mechanisms that would explain this difference are currently unclear, it may be possible that CoMS cells gradually became committed to a steady state high expression level of the KIT deubiquitinating enzyme during their continuous long-term exposure to imatinib. Once the CoMS cells had acquired this property then the addition of imatinib could directly or indirectly trigger activation of this enzyme, resulting in the immediate re-overexpression of KIT and the acquisition of resistance to imatinib.

In conclusion, we have shown that a decrease in KIT ubiquitination by imatinib treatment induces the overexpression of KIT and subsequent imatinib resistance in imatinib sensitive canine MCT cells. Moreover, this decrease in KIT ubiquitination was considered to be associated with the activation of a deubiquitinating enzyme by imatinib. Since canine MCT cells share similar properties with KIT mutation-driven human tumors in terms of sensitivity and the ability to acquire resistance against imatinib, it may be possible that a similar mechanism of KIT overexpression underlies the acquisition of imatinib resistance in some human tumors that are driven by KIT mutation. For a possible transfer of our findings to the human setting, further analysis using canine clinical cases and human cell lines will be needed.

\section{Acknowledgements}

This study was supported in part by a Grant-in-Aid for Scientific Research (no. 15H04601) from the Ministry of Education, Culture, Sports, Science and Technology of Japan.

\section{References}

1. Blay JY, Casali PG, Dei Tos AP, Le Cesne A and Reichardt P: Management of gastrointestinal stromal tumour: Current practices and visions for the future. Oncology 89: 1-13, 2015.

2. Alvarez-Twose I, Matito A, Morgado JM, Sánchez-Muñoz L, Jara-Acevedo M, García-Montero A, Mayado A, Caldas C, Teodósio C, Muñoz-González JI, et al: Imatinib in systemic mastocytosis: A phase IV clinical trial in patients lacking exon 17 KIT mutations and review of the literature. Oncotarget: Jul 19, 2016 (Epub ahead of print). doi: 10.18632/oncotarget.10711.

3. Hodi FS, Corless CL, Giobbie-Hurder A, Fletcher JA, Zhu M, Marino-Enriquez A, Friedlander P, Gonzalez R, Weber JS, Gajewski TF, et al: Imatinib for melanomas harboring mutationally activated or amplified KIT arising on mucosal, acral, and chronically sun-damaged skin. J Clin Oncol 31: 3182-3190, 2013.

4. Gramza AW, Corless CL and Heinrich MC: Resistance to tyrosine kinase inhibitors in gastrointestinal stromal tumors. Clin Cancer Res 15: 7510-7518, 2009.

5. Miranda C, Nucifora M, Molinari F, Conca E, Anania MC, Bordoni A, Saletti P, Mazzucchelli L, Pilotti S, Pierotti MA, et al: KRAS and BRAF mutations predict primary resistance to imatinib in gastrointestinal stromal tumors. Clin Cancer Res 18: 1769-1776, 2012. 
6. Gounder MM and Maki RG: Molecular basis for primary and secondary tyrosine kinase inhibitor resistance in gastrointestinal stromal tumor. Cancer Chemother Pharmacol 67 (Suppl 1): S25-S43, 2011.

7. Debiec-Rychter M, Cools J, Dumez H, Sciot R, Stul M, Mentens N, Vranckx H, Wasag B, Prenen H, Roesel J, et al: Mechanisms of resistance to imatinib mesylate in gastrointestinal stromal tumors and activity of the PKC412 inhibitor against imatinib-resistant mutants. Gastroenterology 128: 270-279, 2005.

8. Miselli FC, Casieri P, Negri T, Orsenigo M, Lagonigro MS Gronchi A, Fiore M, Casali PG, Bertulli R, Carbone A, et al: c-KIT/PDGFRA gene status alterations possibly related to primary imatinib resistance in gastrointestinal stromal tumors. Clin Cancer Res 13: 2369-2377, 2007.

9. Lai S, Wang G, Cao X, Luo X, Wang G, Xia X, Hu J and Wang J: KIT over-expression by p55PIK-PI3K leads to Imatinibresistance in patients with gastrointestinal stromal tumors. Oncotarget 7: 1367-1379, 2016.

10. Irie M, Takeuchi Y, Ohtake Y, Suzuki H, Nagata N, Miyoshi T, Kagawa $Y$ and Yamagami T: Imatinib mesylate treatment in a dog with gastrointestinal stromal tumors with a c-KIT mutation. J Vet Med Sci 77: 1535-1539, 2015.

11. Kobayashi M, Kuroki S, Ito K, Yasuda A, Sawada H, Ono K, Washizu T and Bonkobara M: Imatinib-associated tumour response in a dog with a non-resectable gastrointestinal stromal tumour harbouring a c-KIT exon 11 deletion mutation. Vet J 198: $271-274,2013$

12. London CA, Galli SJ, Yuuki T, Hu ZQ, Helfand SC and Geissler EN: Spontaneous canine mast cell tumors express tandem duplications in the proto-oncogene c-KIT. Exp Hematol 27: 689-697, 1999.

13. Bostock DE: Neoplasms of the skin and subcutaneous tissues in dogs and cats. Br Vet J 142: 1-19, 1986.

14. Bonkobara M: Dysregulation of tyrosine kinases and use of imatinib in small animal practice. Vet J 205: 180-188, 2015

15. London CA, Malpas PB, Wood-Follis SL, Boucher JF, Rusk AW, Rosenberg MP, Henry CJ, Mitchener KL, Klein MK, Hintermeister JG, et al: Multi-center, placebo-controlled, doubleblind, randomized study of oral toceranib phosphate (SU11654), a receptor tyrosine kinase inhibitor, for the treatment of dogs with recurrent (either local or distant) mast cell tumor following surgical excision. Clin Cancer Res 15: 3856-3865, 2009.
16. Kobayashi M, Kuroki S, Tanaka Y, Moriya Y, Kozutumi Y, Uehara Y, Ono K, Tamura K, Washizu T and Bonkobara M: Molecular changes associated with the development of resistance to imatinib in an imatinib-sensitive canine neoplastic mast cell line carrying a KIT c.1523A $>$ T mutation. Eur J Haematol 95: 524-531, 2015.

17. Ishiguro T, Kadosawa T, Mori K, Takagi S, Okumura M and Fujinaga T: Establishment and characterization of a new canine mast cell tumor cell line. J Vet Med Sci 63: 1031-1034, 2001.

18. Jin WL, Mao XY and Qiu GZ: Targeting deubiquitinating enzymes in glioblastoma multiforme: Expectations and challenges. Med Res Rev 37: 627-661, 2017.

19. Reincke M, Sbiera S, Hayakawa A, Theodoropoulou M, Osswald A, Beuschlein F, Meitinger T, Mizuno-Yamasaki E, Kawaguchi K, Saeki Y, et al: Mutations in the deubiquitinase gene USP8 cause Cushing's disease. Nat Genet 47: 31-38, 2015.

20. Masson K, Heiss E, Band $\mathrm{H}$ and Rönnstrand L: Direct binding of Cbl to Tyr568 and Tyr936 of the stem cell factor receptor/c-KIT is required for ligand-induced ubiquitination, internalization and degradation. Biochem J 399: 59-67, 2006.

21. Yee NS, Hsiau CW, Serve H, Vosseller K and Besmer P: Mechanism of down-regulation of c-KIT receptor. Roles of receptor tyrosine kinase, phosphatidylinositol 3'-kinase, and protein kinase C. J Biol Chem 269: 31991-31998, 1994.

22. Kim H, Frederick DT, Levesque MP, Cooper ZA, Feng Y, Krepler C, Brill L, Samuels Y, Hayward NK, Perlina A, et al: Downregulation of the ubiquitin ligase RNF125 underlies resistance of melanoma cells to BRAF inhibitors via JAK1 deregulation. Cell Rep 11: 1458-1473, 2015.

23. Zeng S, Xu Z, Lipkowitz S and Longley JB: Regulation of stem cell factor receptor signaling by Cbl family proteins (Cbl-b/ c-Cbl). Blood 105: 226-232, 2005. 